National Center for Health Statistics; 2009. p. 203.

3. Vicchio M, Della Corte A, De Santos LS, De Feo M, Caianiello G, Scardone M, et al. Tissue versus mechanical prostheses: quality of life in octogenarians. Ann Thorac Surg. 2008;85:1290-5.

4. Sundt TM, Bailey MS, Moon MR, Mendeloff EN, Huddleston CB, Pasque MK, et al. Quality of life after aortic valve replacement at the age of $>80$ years. Circulation. 2000;102(suppl III):70-4.

doi:10.1016/j.jtcvs.2009.04.032

\section{PROXIMAL \\ BRACHIOCEPHALIC ARTERY ACCESS FOR ARTERIAL CANNULATION: VALUE AND TECHNICAL ISSUES \\ To the Editor:}

I read with great interest the report by Roy and colleagues ${ }^{1}$ on the rapid resuscitation of an infant with rescue extracorporeal life support (ECLS) with simple access through the innominate artery. In their case, the stump of a $3.0-\mathrm{mm}$ polytetrafluoroethylene (PTFE) graft anastomosed to the innominate artery during the initial stage I Norwood (Sano) procedure was rapidly recannulated to provide arterial access for ECLS. This case provides yet another good example of the utility, simplicity, and safety of proximal brachiocephalic artery access for arterial cannulation. At my institution, we have used the same strategy for arterial cannulation during the Norwood procedure for a number of years and have found it similarly useful in a number of ways. Importantly, there have been no technical complications related to the use of this technique. Specifically, there have been no early or late innominate artery stenoses or occlusions. In fact, the technique has been so useful that it has been adopted for many types of cases requiring aortic work, such as interrupted aortic arch, Damus-Kaye-Stansel, coarctation and ventricular septal defect.

I also suspect that this technique may be of further use in cases in which transverse aortic size is borderline and coronary perfusion could be compromised by direct aortic cannulation. At our institution, we have had a single case of d-transposition of the great arteries with particularly complicated coronary anatomy in which the switch from direct aortic cannulation to innominate artery cannulation through a PTFE conduit resulted in rapid hemodynamic recovery and separation from ECLS. In fact, this method of arterial cannulation has since been used for nearly all arterial switch procedures, with no mortality and no use of ECLS in a small series of patients. I and my colleagues have recently set out to investigate scientifically whether there is some benefit to this method of cannulation in neonates with regard to coronary perfusion.

I have a few technical questions for Roy and colleagues regarding the use of this technique. First, what is the ultimate fate of the PTFE when the chest is closed? How long a segment do you ultimately leave in the chest when it is closed? Do you allow the PTFE to lie on top of the innominate vein or tuck it in underneath the vein, next to the aorta? At my institution, we have been concerned about the possibility of creating a scissoring effect on the vein between the PTFE and the aorta and thus have tucked it beneath the vein but still worry about vein occlusion. Additionally, what steps do you take to clear the PTFE of clot and debris before rapid cannulation? We attempt to avoid the formation of clot by multiply clipping the graft proximally and distally. Finally, has your group ever considered using a slightly larger PTFE graft and reusing it later for the Glenn procedure if cardiopulmonary bypass is necessary?

I greatly appreciate this short report by Roy and colleagues ${ }^{1}$ regarding this extremely useful technique. I not only congratulate them on the survival of their patient but also applaud the dissemination of information with so much practical importance.

Benjamin B. Peeler, MD Associate Professor of Surgery and

\section{Pediatrics \\ University of Virginia School of Medicine \\ Surgical Director, Virginia Children's \\ Heart Center \\ Charlottesville, $\mathrm{Va}$}

\section{Reference}

1. Roy N, Rebeyka IM, Atallah, Ross DB. Rapid extracorporeal life support rescue in patients undergoing the Norwood procedure. J Thorac Cardiovasc Surg. 2009;137:765-6.

doi:10.1016/j.jtcvs.2009.04.053

\section{Reply to the Editor:}

We appreciate your comments and agree that innominate artery cannulation facilitates aortic arch surgery in the newborn and may be useful with other neonatal cardiac repairs, especially when the ascending aorta is diminutive.

We typically use delayed sternal closure after stage 1 repair of the hypoplastic left heart and trim the graft to about $4 \mathrm{~cm}$ in length after clipping the proximal end immediately adjacent to the innominate artery (see Figure 1 in our article $\left.{ }^{1}\right)$.The graft is anastomosed to the innominate artery cephalad to the innominate vein and sits in the upper mediastinum, well above the vein. At the time of delayed sternal closure, the graft is trimmed to within several millimeters of the occluding clips.

If emergency cannulation for extracorporeal life support is required, the shunt is stripped with forceps and then flushed with heparinized saline with the proximal clips in place. The clips are then removed and retrograde flow through the shunt established. An 8F BioMedicus cannula (Medtronic BioMedicus, Eden Prairie, Minn) is then inserted in the graft and secured with 2-0 silk, before connection to the extracorporeal life support circuit.

We have not used the same graft for cannulation at the time of the cavopulmonary shunt and prefer direct aortic cannulation. 